\title{
Mixed Gonadal Dysgenesis with an Ovarian Germ Cell Tumor in a Filipino Adolescent
}

\author{
Marie Julianne C. Racoma, MD, ${ }^{1}$ Julianne Keane M. Pascual, MD, ${ }^{2}$ \\ Edsel Allan G. Salonga, $\mathrm{MD}^{3}$ and Mary Ann R. Abacan, MD, MSc ${ }^{1}$ \\ ${ }^{1}$ Division of Clinical and Metabolic Genetics, Department of Pediatrics, Philippine General Hospital, University of the Philippines Manila \\ ${ }^{2}$ Department of Pediatrics, Philippine General Hospital, University of the Philippines Manila \\ ${ }^{3}$ Institute of Human Genetics, National Institutes of Health, University of the Philippines Manila
}

\begin{abstract}
We present a case of a 13-year-old Filipino patient with ambiguous genitalia, randomly assigned as female at birth and was subsequently reared as such. Starting at 10 years of age, secondary male sexual characteristics emerged. However, medical consultation was sought only when the patient developed a gradually enlarging and painful abdominal mass. After cytogenetic studies, we are able to identify the case as Disorder of Sex Development, 45,X/46,X,der(Y) mosaicism, mixed gonadal dysgenesis. Further imaging, laparotomy and histopathology, revealed that the abdominopelvic mass was from the right ovary, composed of immature teratoma. Ductus deferens and epididymis were also identified within the right ovary. At the time of presentation, the patient was already experiencing gender dysphoria. This report not only highlights the wide spectrum of phenotypes in mixed gonadal dysgenesis, but also stresses the importance of proper gender assignment during the newborn period in patients with disorders of sex development.
\end{abstract}

Key Words: disorder of sexual development, ambiguous genitalia, mixed gonadal dysgenesis, mosaicism, 45,X/46,X,der(Y) karyotype, immature teratoma, gender dysphoria

\section{INTRODUCTION}

$2^{\text {nd }}$ Place, Fellows Case Report Category. Poster presented at the Department of Pediatrics, Research Week on October 2019, University of the Philippines-Philippine General Hospital.

Poster presented at the, Asia-Pacific Conference on Human Genetics on November 2019, Manila, Philippines.

Corresponding author: Marie Julianne C. Racoma, MD Department of Pediatrics

Philippine General Hospital

University of the Philippines Manila,

Taft Avenue, Ermita, Manila 1000

Email:mcracoma@up.edu.ph
Disorder of sex development (DSD) is defined as a group of congenital conditions characterized by atypical chromosomal, gonadal, and anatomic sex development. ${ }^{1}$ Knowledge of these disorders is imperative because they are considered to be one of the most common birth defects worldwide, comprising 7\%. Around 1 in 4500 babies are born with ambiguous genitalia. ${ }^{2}$

The broad term "DSD" includes common and uncommon entities and are further classified based on the primary genetic defect. Mixed gonadal dysgenesis (MGD) results from a numerical sex chromosome abnormality leading to abnormal gonadal development. It has a wide spectrum of clinical manifestations, but commonly presents as ambiguous genitalia. The karyotype is mostly mosaic, and the presence of $45, X$ cell lines is frequently associated with $\mathrm{Y}$ chromosome rearrangements, as in our case.

This report will help elucidate the highly variable manifestations of MGD by presenting a unique case. It will also emphasize the importance of early identification of this disorder to be able to provide timely and holistic management of patients with DSD.

We present a case of MGD with mosaicism diagnosed with a rare malignant germ cell tumor (GCT) in a Filipino adolescent who was not evaluated for ambiguous genitalia until the development of a painful abdominal mass from the tumor. 


\section{CASE REPORT}

\section{History of Present IIIness}

A 13-year-old patient, reared as female, was seen at the Philippine General Hospital and referred to the Clinical Genetics service for evaluation of an ambiguous genitalia and enlarging abdominal mass. Informed consent and assent for the publication of medical data and photographs were taken from the parents and patient, respectively. The patient was born to a then 19 -year-old primigravid via spontaneous vaginal delivery at full term. The prenatal course was unremarkable. The patient is the only child born to a healthy non-consanguineous couple of Filipino descent. At birth, the patient was noted to have a phallic-like structure surrounded by bilateral rugated labioscrotal folds. Despite the genital ambiguity, she was considered female and was subsequently raised as such. Newborn screening and further investigations were not done.

At 10 years old, the patient started to develop secondary male characteristics, such as thickened pubic and axillary hair, facial hair, enlarging laryngeal prominence, and deepening of voice. Breast development and menarche did not occur.

At 12 years old, the patient complained of rightsided abdominal pain associated with early satiety and a gradually enlarging right lower quadrant mass which prompted medical consult.

\section{Clinical and Laboratory Findings}

The patient's height was $140.5 \mathrm{~cm}$ (less than the $3^{\text {rd }}$ percentile) and weight $34.5 \mathrm{~kg}$ (less than the $3^{\text {rd }}$ percentile), with a blood pressure of $100 / 60 \mathrm{mmHg}$. Aside from short stature, other features associated with Turner syndrome, such as webbed neck, low nuchal hairline, broad chest, lymphedema, and cardiac and renal anomalies, were not appreciated. The patient had thick facial and axillary hair with apparent laryngeal prominence. Pigmentary mosaicism of the hyperpigmented type was noted over the chest and abdomen. Breast development was at Tanner Stage I. Abdomen was globular with a palpable tender, fixed, abdomino-pelvic mass measuring $20 \mathrm{~cm} \times 23 \mathrm{~cm} \times 10 \mathrm{~cm}$ (Figure 1). Initial complete blood count, serum chemistry and urinalysis were normal. Chest $\mathrm{x}$-ray and $12 \mathrm{~L}-\mathrm{ECG}$ did not reveal any abnormalities.

\section{Genital Organs}

Examination of the external genitalia showed pubic hair development at Tanner Stage III, a phallus that measures $7 \mathrm{~cm}$ from the base, with ventral hypospadias and rugated labioscrotal folds without palpable mass. External masculinization score (EMS) was 5 out of $12 .{ }^{3}$ The patient had a blind vaginal sac, $0.5 \mathrm{~cm}$ deep (Figure 2). Transrectal and abdominal ultrasound revealed an abdominopelvic mass, suggestive of a malignant left ovarian new growth. There was also note of uterine hypoplasia with thin endometrium.
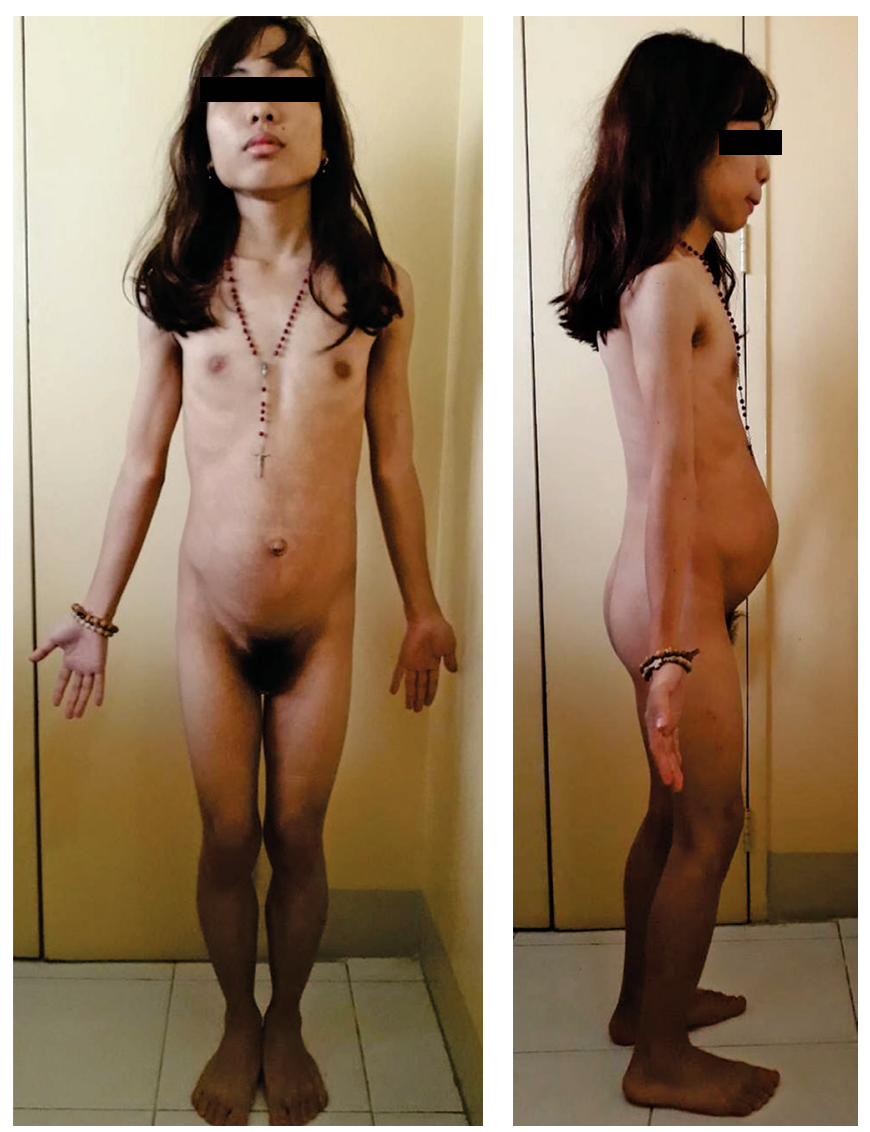

Figure 1. Case of mixed gonadal dysgenesis with mosaicism in a 13-year-old patient.
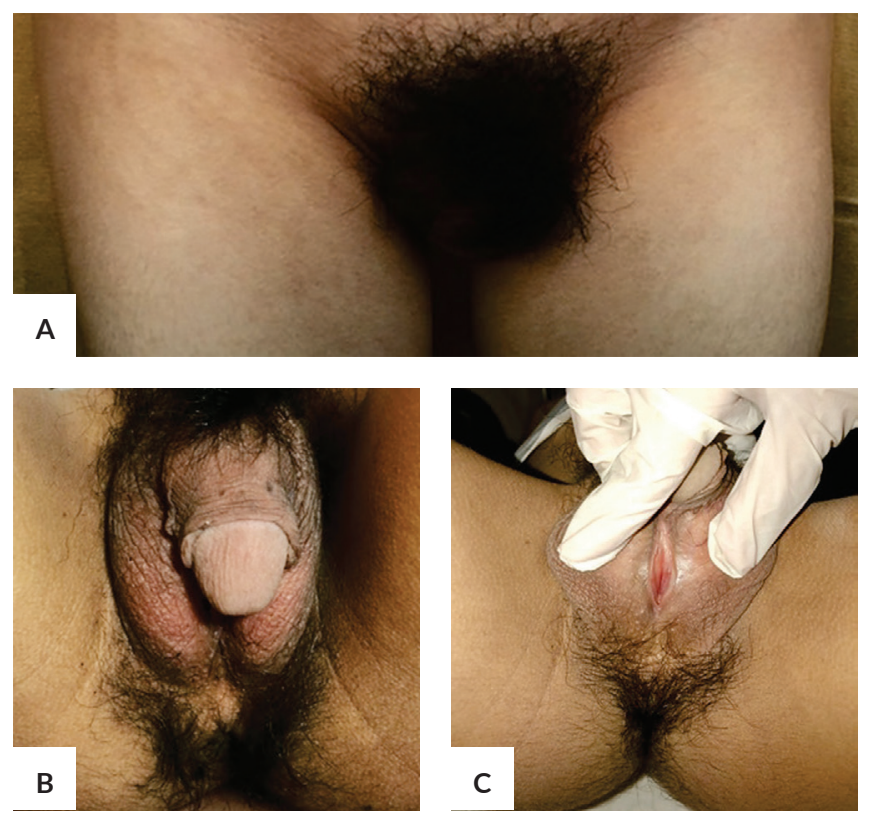

Figure 2. Tanner Stage III for pubic hair (A) with a $7 \mathrm{~cm}$ phallus with ventral hypospadias and rugated labioscrotal folds without palpable mass $(B)$, and a blind vaginal sac, $0.5 \mathrm{~cm}$ deep (C). 
Whole abdominal CT scan showed a complex abdomino-pelvic mass with cystic and calcific components which may be consistent with a teratoma. There was a tubular structure in the anterior pelvic region representing the penile shaft with adjacent prominent soft tissue on both sides, but no distinct testicle in the hypoplastic scrotal sac.

\section{Chromosomal Analysis}

Initial identification of the karyotype was performed using G-banding method. The chromosomal analysis revealed two abnormal cell lines present in the 50 cells that were examined. The first cell line, seen in 26 cells, showed an abnormal female chromosome complement with loss of one $\mathrm{X}$ chromosome, resulting to monosomy X. The second cell line, seen in 24 cells, showed an abnormal female chromosome complement with one copy of the $\mathrm{X}$ chromosome and one small unidentified marker chromosome. FISH analysis identified the marker chromosome as derivative of $\mathrm{Y}$ chromosome using the $\mathrm{Y}$ alpha-satellite probe DY23. This was reported as 45,X/46, $\mathrm{X}, \operatorname{der}(\mathrm{Y})$ (Figure 3).

\section{Endocrinologic Tests and Tumor Markers}

All levels were determined prior to laparotomy. Baseline serum cortisol (263.54 nmol/L; normal value: 101.2-535.7) and estradiol levels $(79.98 \mathrm{pmol} / \mathrm{L}$; normal value: 40.37161.48) were within normal range. Testosterone was elevated at $8.88 \mathrm{nmol} / \mathrm{L}$ (normal value: $0.31-3.78$ [premenopausal]). Thyroid function test was normal with FT4 of $15.297 \mathrm{pmol} / \mathrm{L}$ (normal value: 8.8-33) and TSH of $3.491 \mathrm{uIU} / \mathrm{mL}$ (normal value: $0.27-3.75)$.

Total beta-HCG was elevated at $29.73 \mathrm{mIU} / \mathrm{mL}$ (normal value: $<5$ [non=pregnant]), as well as AFP at 71.10 $\mathrm{IU} / \mathrm{mL}$ (normal value: $0.74-7.3$ ). Other tumor markers were all elevated, as follows: CA $125 \mathrm{II}$ of $319.7 \mathrm{U} / \mathrm{mL}$ (normal value: <35), CA $19-9$ of $>1200 \mathrm{U} / \mathrm{mL}$ (normal value: <37), and CEA $5.12 \mathrm{ng} / \mathrm{mL}$ (normal value: $<5$ ).

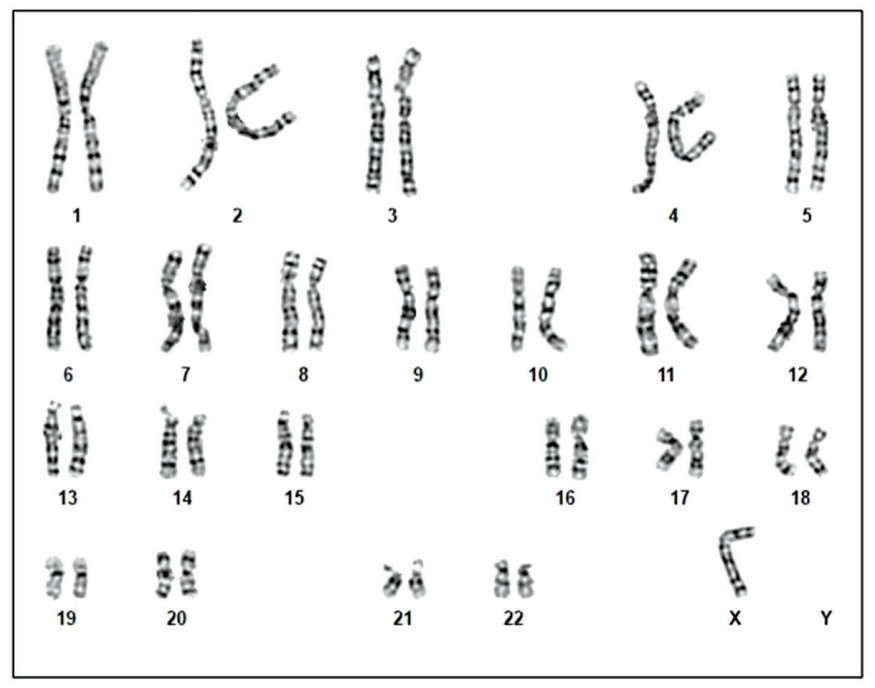

A

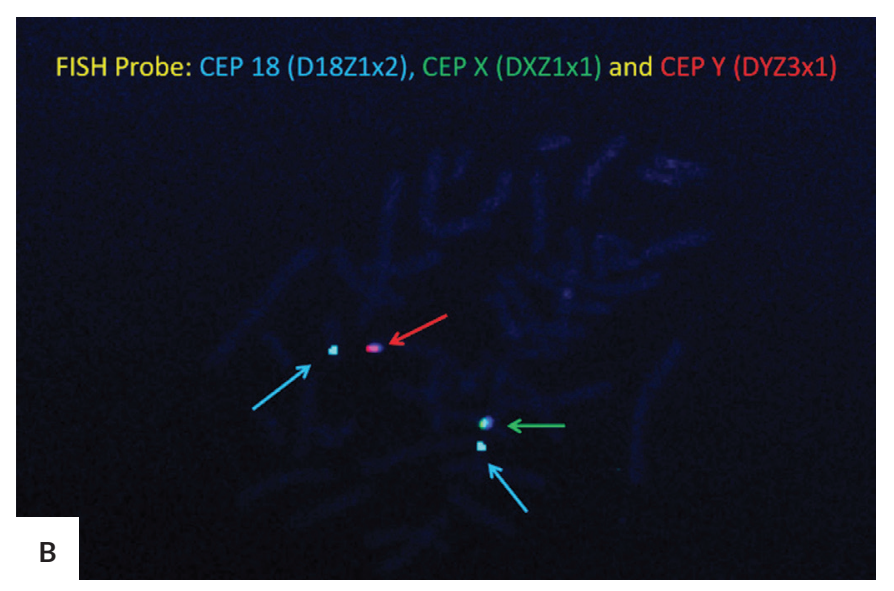

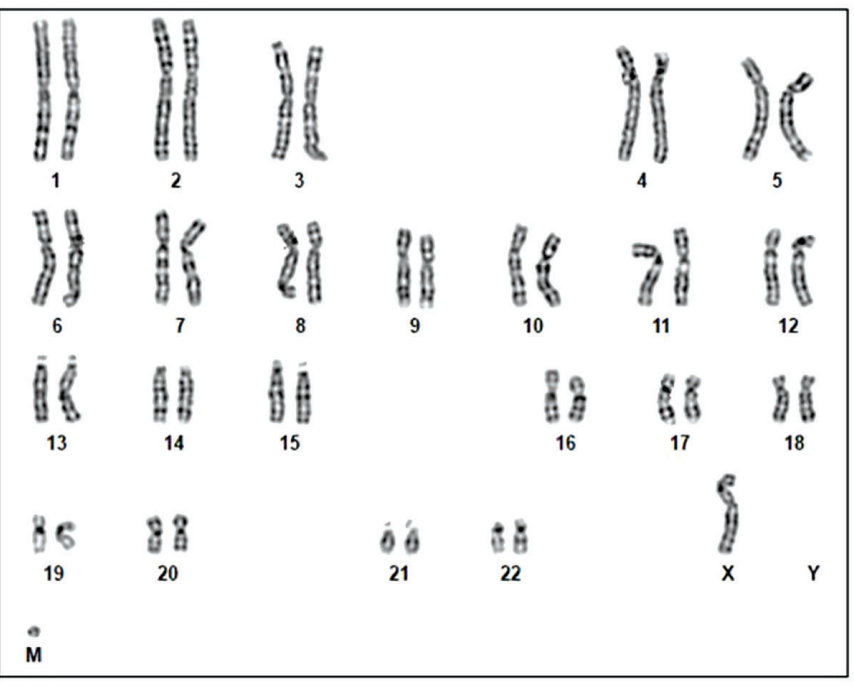

Karyotype: 46,X,mar
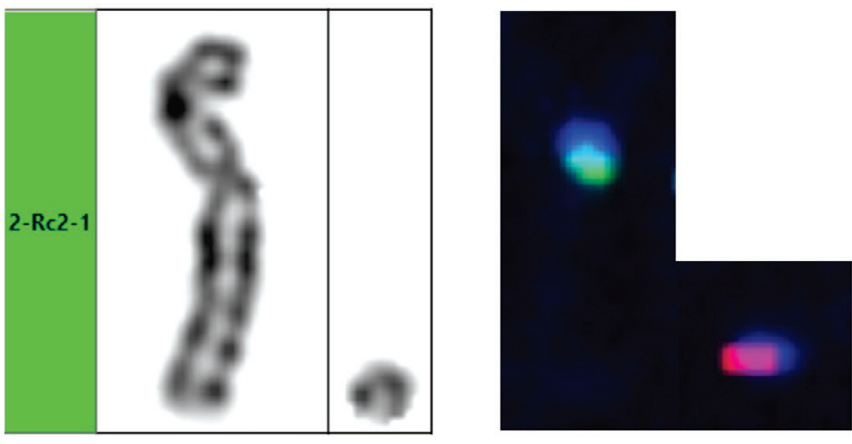

C

Figure 3. Abnormal karyotype: Mosaic monosomy $X$ with cell line with marker chromosome, 45,X[26]/46,X,+mar[24] (A). ISCN FISH: Mosaic monosomy $X$ with a male cell line with a derivative $Y$ chromosome, 45, $X[26] / 46, X,+\operatorname{mar}[24]$.ish der(Y) (DYZ3+) (B). Zoomed view comparing partial karyotype of $X$ with marker and FISH identifying the $X$ and marker as $Y(C)$. 


\section{Surgical Management}

The patient underwent exploratory laparotomy, total hysterectomy with bilateral salphingo-oophorectomy, pelvic lymph node dissection, para-aortic lymph node sampling, infracolic omentectomy and peritoneal biopsy. Intraoperatively, the right ovary was noted to have been converted to a $17 \mathrm{~cm} \times 20 \mathrm{~cm} \times 12 \mathrm{~cm}$ solid mass with necrotic areas, with presence of skin, hair, cartilage and sebum. On histopathologic examination, ductus deferens and epididymis were identified in the right ovary. The uterus measured 3.5 $\mathrm{cm} \times 3.5 \mathrm{~cm} \times 1 \mathrm{~cm}$ with smooth serosal and endometrial surfaces. There was also note of a left ovarian streak measuring $1 \times 2 \mathrm{~cm}$ and with a corresponding fallopian tube (Figure 4).

Final histopathologic diagnosis of the right ovary was: Immature teratoma, Norris Grade 3,16 cm in greatest dimension. Immunohistochemistry showed that alpha fetoprotein (AFP) was negative in the cells of interest. Postoperatively, the plan was to start standard postoperative adjuvant chemotherapy with bleomycin-etoposide-cisplatin.

\section{Psychosocial Issues}

At the time of presentation, the patient was an $8^{\text {th }}$ grade student, with signs of gender dysphoria. The patient identified as female with sexual interest towards the male sex; but due to apparent masculinization over the years, the patient had already started to develop issues on gender identity and became more socially withdrawn. The patient denied any suicidal ideations, but often expressed desire to undergo hormone therapy for feminization of features. The patient was referred to Child Psychiatry and Adolescent Medicine services for psychosocial support. Familial dynamics were also to be addressed, as the mother and stepfather were uncomfortable dealing with the patient's condition.

\section{DISCUSSION}

Disorders of sexual development comprise a group of congenital conditions characterized by incongruence in chromosomal, gonadal, and anatomic sex development. ${ }^{4}$ The umbrella term "DSD" includes variable entities, from the common to the uncommon, resulting from atypical sexual differentiation. It is further subclassified into three distinct groups, published by the Lawson Wilkins Pediatric Endocrine Society and the European Society for Paediatric Endocrinology, and is based on the primary genetic defect: 1 ) Sex chromosome DSD, 2) 46,XY DSD, and 3) 46,XX DSD. Karyotyping is an initial test that allows for the classification of the disorder. Under this new nomenclature, we classify our patient as having sex chromosome DSD, particularly under 45X/46XY mixed gonadal dysgenesis (MGD). ${ }^{4}$

Normal differentiation of female and male genitalia happens between $6-8^{\text {th }}$ week age of gestation with the formation of the bipotential gonad. It will then differentiate into either testicular or ovarian tissue, depending on whether the egg, which always carries an $\mathrm{X}$ chromosome, will be
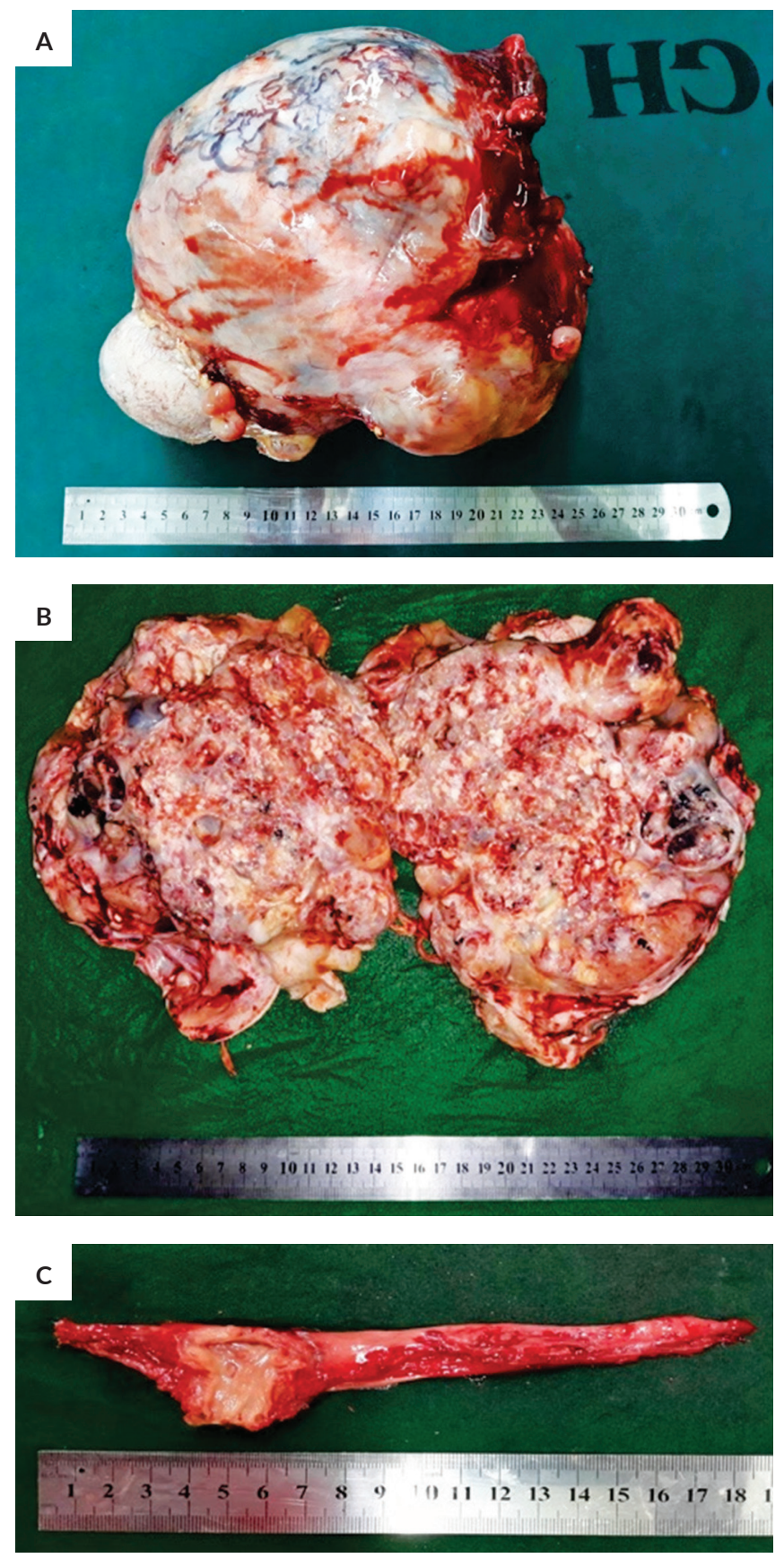

Figure 4. Gross (A) and cut section (B) of the right ovarian mass. Left ovarian streak with fallopian tube (C).

fertilized by a sperm with either an $\mathrm{X}$ or $\mathrm{Y}$ chromosome. For the activation of these sexual differentiation pathways to occur, several transcription factors that regulate the expression of tissue-specific genes and signaling molecules must be expressed. Male differentiation is initiated by the $S R Y$ (sex-determining region Y) encoded in the short arm of $Y$ chromosome. This gene will produce $S R Y$ protein that allows for the development of the bipotential gonad into testes, via a genetic cascade of expression of NR5A1, SOX9, and $D A X 1$, among others. While in feminization $(\mathrm{XX})$, there 
is absence of $S R Y$ gene, thus activating a cascade of different transcription factors, including WNT4, DAX1, and RSPO1, that ultimately leads to development of the bipotential gonads into ovaries. ${ }^{5}$ It is important that their gene expressions have to be balanced - in that the genes and their products have to be expressed in the right amounts, at the right time, and in the right place in the developing embryo. Any mutations or imbalance in their expression will lead to DSDs. ${ }^{2}$

Gonadal dysgenesis is a subset of DSD characterized by incomplete or defective formation of the gonads caused by either structural or numerical anomalies of the sex chromosomes or mutations in the genes involved in the development of the gonad. Patients with MGD often occur as mosaic - the presence of two or more distinct cell lines in an individual. ${ }^{2}$ The classic form of MGD is associated with $45, \mathrm{X} / 46, \mathrm{XY}$ mosaicism. As reported, the phenotype associated with this mosaicism ranges from infants with ambiguous genitalia to females with Turner syndrome stigmata, hypospadias, and gonadal dysgenesis, as well as sterile male phenotypes. However, features cannot be accurately predicted by this peripheral blood study alone, as mosaicism varies in different somatic tissues. ${ }^{6} \mathrm{~A}$ common mechanism for the $45, \mathrm{X} / 46, \mathrm{XY}$ mosaic variant is the loss of structural abnormal $\mathrm{Y}$ material arising via the anaphase lag during mitosis in the zygote. Although $\mathrm{Y}$ chromosomal abnormalities and interchromosomal rearrangements with loss of structural abnormal $\mathrm{Y}$ material are sometimes seen, there may be other combinations of chromosome patterns at work. As a result, MGD patients have an underdevelopment of gonads, known as "dysgenetic" or "streak" gonads. Dysgenetic gonads are characterized by variable degrees of immaturity or dysfunction, including underproduction of sex hormones. ${ }^{7}$

The variability of the MGD phenotype depends on the presence or absence of the testis-determining $S R Y$ gene, on the degree of mosaicism, and the tissue distribution of the $45, \mathrm{X}$ cell line. Internal genitalia may have remnants of the Müllerian duct structures, such as rudimentary uterus and fallopian tube, because of anti-Müllerian hormone $(\mathrm{AMH})$ deficiency or insensitivity of the paramesonephric ducts to $\mathrm{AMH} .{ }^{8}$ In mosaic patients, the gonads are usually asymmetrical, with a dysgenetic testis on one side, and a streak gonad on the other side. ${ }^{8}$

As mentioned, the karyotype is mostly mosaic, and the presence of $45, X$ cell lines is frequently associated with $\mathrm{Y}$ chromosome rearrangements, commonly dicentric and ring $\mathrm{Y}$ chromosomes., ${ }^{9,10}$ In a review by Johansen et. al., which studied 25 patients with 45,X/46,XY mosaicism, it was reported that eight of the participants (32\%) had structural rearrangements of the $\mathrm{Y}$ chromosome, as follows: five patients with isodicentricism of the $\mathrm{Y}$ chromosome, two patients with deletions of the $\mathrm{Y}$ chromosome, and one patient with translocation between the $\mathrm{Y}$ chromosome and chromosome $22 .{ }^{11}$

Another important observation from the Johansen study was that even male patients with ambiguous genitalia have a good chance of Leydig cell function and thus enter spontaneous puberty. However, their fertility potential remains uncertain. This implies that, although the phenotypes of MGD patients vary greatly, the EMS does not always reflect the true masculinization of the gonad. And while some phenotypically male patients might still present with growth retardation even after spontaneous pubertal onset, they appear to respond well to growth hormone treatment. ${ }^{11}$ In our patient, despite the low EMS of $5 / 12$, there was spontaneous development of secondary male characteristics. This implies normal testicular function that might later be supported by hormone-replacement therapy (HRT). However, our case presents a unique dilemma since the patient was already reared and had identified as female, and as such would not be agreeable to testosterone therapy.

As a group, patients with DSD are at an increased risk for gonadal malignancy, as there is a well-established relationship between gonadoblastoma development, dysgenetic gonads, and the presence of Y-derived material. ${ }^{12}$ Researchers speculated that some factors involved in the growth of dysgenetic gonads might be the same as those involved in the development of gonadoblastoma. ${ }^{13,14}$ In another report, it was theorized that there is a gonadoblastoma locus in the $\mathrm{Y}$ chromosome that acts as a yet unidentified oncogene. ${ }^{15}$

Although there are varying reports on the estimated tumor risk, the presence of MGD with a $\mathrm{Y}$ chromosome places the patient at the highest risk for gonadal malignancy of up to $35 \%$, warranting gonadectomy at the time of diagnosis.,16 In general, the most common malignant germ cell neoplasm associated with gonadal dysgenesis is gonadoblastoma; while others including dysgerminomas, yolk-sac tumors, and teratomas are rare but can occur. ${ }^{17}$ In our case, evaluation for ambiguous genitalia was only initiated during the adolescent period, when the patient developed a painful abdominal mass. The untimely medical attention led to the development of ovarian GCT consisting of immature teratoma. Grading of this relatively rare neoplasm is based on the proportion of immature neuroepithelial component. As of 2019, there is no consensus regarding therapeutic management.In a study by Shinkai et al., with a median followup of seven years, event-free survival was reported among seven pediatric patients. ${ }^{18}$ Post-operative chemotherapy was also deemed unnecessary even for higher grade teratomas, and those with low AFP levels, as supported by Pashankar et al. ${ }^{19}$ Their pooled analysis on adjuvant chemotherapy for immature teratomas revealed that chemotherapy did not decrease relapses in the pediatric cohort, even among highergrade neoplasms. In this paper, 13 of the 44 patients enrolled had microscopic foci of a yolk sac tumor. Their event-free survival rate was $97.7 \%$ after 4 years. Thus, surgery alone was curative in cases of completed resected tumors, regardless of grade or presence of yolk sac tumor. Nevertheless, long-term follow-up is recommended for surveillance of ipsilateral lesions or contralateral tumor developments. 
The delay in diagnosis and management not only had negative health implications, but also resulted to psychosocial issues in our patient. At the time of presentation, there were already signs of gender dysphoria - "the feeling of being the opposite gender to which one was born or assigned and wanting to live as that opposite gender" ${ }^{1,3,20}$ This stresses the integral role of psychosocial support in our patient's management in order to promote positive adaptation. Particular attention to mental health should be given during the adolescent period, as this is a difficult time even for children without DSD. Patients should be referred to a psychologist to provide them the opportunity to discuss their thoughts about gender identity, sexuality, sexual orientation, and relationships in a safe and nurturing environment. ${ }^{1,3,20}$

Because of the complex nature of the disorder, a multidisciplinary team of experts is required to address the medical needs and the psychosocial issues that the DSD patient and the family may encounter. The geneticist is an integral part of the team to perform proper genetic diagnostic tests early on. Once a genetic diagnosis has been confirmed, genetic counseling for the family and child as well as assessment for the need to evaluate other family members should be provided. ${ }^{21}$

This report not only highlights the wide spectrum of phenotypes in MGD, but also emphasizes the importance of early identification and management of patients with ambiguous genitalia. An important milestone in every pregnancy is the ascertainment of the baby's sex, whether prenatally or at birth. Thus, genital ambiguity is not only considered as a complex medical emergency, but as a social emergency as well. There is a need for pediatricians to be knowledgeable of this disorder since it is not uncommon. Sex assignment should be done during the newborn period. It should take into account several crucial factors in the process of sex assignment, such as diagnosis, karyotype, appearance of the external genitalia, type of gonads present, need for surgical interventions to provide consistency with the sex of rearing, use of hormonal therapy, potential for fertility, parental wishes, and parental cultural beliefs. ${ }^{1}$ This is followed by timely gonadectomy, if applicable. This holistic approach will minimize long-term physical and psychological risks and allow for these patients to develop into well-adjusted and productive members of society.

\section{Statement of Authorship}

MJR and JMP conceived the original idea and wrote the manuscript. All authors discussed the contents, contributed to the final manuscript and approved the final version submitted.

\section{Author Disclosure}

All authors declared no conflicts of interest.

\section{Funding Source \\ Self-funded.}

\section{REFERENCES}

1. Moshiri M, Chapman T, Fechner P, Dubinsky T, Shnorhavorian $\mathrm{M}$, Osman S, et al. Evaluation and management of disorders of sex development: multidisciplinary approach to a complex diagnosis. RadioGraphics. 2012;32(6):1599-1618.

2. Nussbaum R, McInnes R, Willard H, Hamosh A. Thompson \& Thompson Genetics in Medicine. Philadelphia, PA: Elsevier; 2016.

3. Ahmed S, Rodie M. Investigation and initial management of ambiguous genitalia. Best Practice \& Research Clinical Endocrinology \& Metabolism. 2010;24(2):197-218.

4. Hughes I, Houk C, Ahmed S, Lee P. Consensus statement on management of intersex disorders. Journal of Pediatric Urology. 2006;2(3):148-62.

5. García-Acero M, Moreno-Niño O, Suárez-Obando F, et al. Disorders of sex development: Genetic characterization of a patient cohort. Mol Med Rep. 2020;21(1):97-106. doi:10.3892/mmr.2019.10819.

6. Gersen S, Keagle MB. The Principles of Clinical Cytogenetics. 3rd edition. New York: Springer, 2013.

7. McCann-Crosby B, Mansouri R, Dietrich JE, McCullough LB, Sutton VR, Austin EG, et al. State of the art review in gonadal dysgenesis: challenges in diagnosis and management. Int J Pediatr Endocrinol. 2014;2014(1):4. doi: 10.1186/1687-9856-2014-4. Epub 2014 Apr 14. PMID: 24731683; PMCID: PMC3995514.

8. Fernandez R, Pasaro E. Molecular analysis of an $\operatorname{idic}(\mathrm{Y})$ (qter $\rightarrow$ p11.32::p11.32 $\rightarrow$ qter) chromosome from a female patient with a complex karyotype. Genet Mol Res. 2006; 5:399-406.

9. Öçal G. Current concepts in disorders of sexual development. Journal of Clinical Research in Pediatric Endocrinology. 2011;3(3):105-114.

10. El-Sherbiny M. Disorders of sexual differentiation: I. Genetics and pathology. Arab Journal of Urology. 2013;11(1):19-26.

11. Johansen ML, Hagen C, Rajpert-De Meyts E, Kjærgaard S, Petersen B, Skakkebæk N et al. 45,X/46,XY Mosaicism: phenotypic characteristics, growth, and reproductive function-a retrospective longitudinal study. The Journal of Clinical Endocrinology \& Metabolism. 2012;97(8):E1540-E1549.

12. Ai-Xia Liu, Hai-Yan Shi, Zhe-Jun Cai, Aiping Liu, Dan Zhang, He-Feng Huang, et al. Increased risk of gonadal malignancy and prophylactic gonadectomy: a study of 102 phenotypic female patients with Y chromosome or Y-derived sequences,.Human Reproduction. 2014;29(7):1413-1419, https://doi.org/10.1093/humrep/deu109.

13. Jørgensen N, Muller J, Jaubert F, Clausen OP, Skakkebaek NE. Heterogeneity of gonadoblastoma germ cells: similarities with immature germ cells, spermatogonia and testicular carcinoma in situ cells, Histopathology, 1997;30:177-86.

14. Mazzanti L, Cacciari E, Bergamaschi R, Tassinari D, Magnani C, Perri A, Scarano E, Pluchinotta V. Pelvic ultrasonography in patients with Turner syndrome: age-related findings in different karyotypes. J Pediatr 1997; 131:135-40.

15. Lahn BT, Page DC. Functional coherence of the human Y chromosome. Science 1997;278:675-80.

16. Cools M, Stoop H, Kersemaekers A, Drop S, Wolffenbuttel K, Bourguignon J, et al. Gonadoblastoma arising in undifferentiated gonadal tissue within dysgenetic gonads. The Journal of Clinical Endocrinology \& Metabolism. 2006;91(6):2404-13.

17. Piazza M, Urbanetz A. Germ Cell Tumors in Dysgenetic Gonads. 2020.

18. Shinkai T, Masumoto K, Chiba F, Shirane K, Tanaka Y, Aiyoshi $\mathrm{T}$, et al. Pediatric ovarian immature teratoma: histological grading and clinical characteristics. Journal of Pediatric Surgery. doi.org/ 10.1016/j.jpedsurg.2019.04.037 0022-3468

19. Pashankar F, Hale J, Dang, H, Kralio, M, Brady W, Rodriguez-Galindo $\mathrm{C}$, et al. Is adjuvant chemotherapy indicated in ovarian immature teratomas? A combined data analysis from the Malignant Germ Cell Tumor International Collaborative. Cancer; 2016(2):230-7.

20. Kim K, Kim J. Disorders of Sex Development. Korean Journal of Urology. 2012;53(1):1

21. Gomez-Lobo V. 2014. Multidisciplinary care for individuals with disorders of sex development 